OPEN ACCESS

Edited by:

Semih Esin,

University of Pisa, Italy

Reviewed by:

Mervi Gürsoy,

University of Turku, Finland

Giuseppantonio Maisetta,

University of Pisa, Italy

*Correspondence:

Casey M. Gries

Casey.Gries@medsch.ucr.edu

Specialty section:

This article was submitted to

Bacteria and Host,

a section of the journal

Frontiers in Cellular and Infection

Microbiology

Received: 18 June 2020 Accepted: 10 September 2020

Published: 15 October 2020

Citation:

Gries CM, Rivas Z, Chen J and Lo DD (2020) Intravital Multiphoton

Examination of Implant-Associated

Staphylococcus aureus Biofilm

Infection.

Front. Cell. Infect. Microbiol.

10:574092

doi: 10.3389/fcimb.2020.574092

\section{Intravital Multiphoton Examination of Implant-Associated Staphylococcus aureus Biofilm Infection}

\author{
Casey M. Gries*, Zuivanna Rivas, Justin Chen and David D. Lo \\ Division of Biomedical Sciences, School of Medicine, University of California, Riverside, Riverside, CA, United States
}

Bacterial infections associated with implanted medical devices represents a healthcare crisis due to their persistence, antibiotic tolerance, and immune avoidance. Indwelling devices are rapidly coated with host plasma and extracellular matrix proteins which can then be exploited by bacterial pathogens for adherence and subsequent biofilm development. Our understanding of the host-pathogen interface that determines the fate of biofilm-mediated infections is limited to the experimental models employed by laboratories studying these organisms. Current in vivo models of biofilm-mediated infection, while certainly useful, are typically limited to end-point analyses of bacterial burden enumeration, immune cell profiling, and cytokine/chemokine analysis. Thus, with these models, the complex, real-time assessment of biofilm development and innate immune cell activity remains imperceptible. Here, we describe a novel murine biofilm infection model employing time-lapse intravital multiphoton microscopy which permits concurrent and real-time visualization of Staphylococcus aureus biofilm formation and immune cell activity. Using cell tracking, we found that $S$. aureus biofilms impede neutrophil chemotaxis, redirecting their migration patterns to prevent biofilm invasion. This approach is the first to directly examine device-associated biofilm development and host-pathogen interactions and will serve to both further our understanding of infection development and help reveal the effects of future antibiofilm treatment strategies.

Keywords: multiphoton microscopy, medical device infection, Staphylococcus aureus, biofilm, innate immunity

\section{INTRODUCTION}

Bacterial biofilm infections remain a significant healthcare problem worldwide. Infection risk is substantially increased in the presence of an implanted medical device, such as an orthopedic prosthesis, electronic cardiac device, artificial heart valve, or indwelling catheter (Costerton et al., 2005; Tande and Patel, 2014; Arciola et al., 2018). Over 25\% of nosocomial infections are associated with an implanted medical device (Magill et al., 2014), and the incidence of infected hip and knee arthroplasty rates are expected to rise (Kurtz et al., 2007; Tande and Patel, 2014). The current standard-of-care involves surgical debridement and, if necessary, a two-step removal of the infected hardware including placement of a temporary spacer, 4-8 weeks of parenteral antimicrobial therapy, followed by insertion of a new device. This long and debilitating process is associated with significant patient morbidity and financial burden, often exceeding $\$ 90,000$ per infection (Kurtz et al., 2012). In addition, prior device-associated infection increases patient risk for 
infection relapse. Thus, an urgent need exists for novel approaches to prevent device-associated infection and/or facilitate biofilm eradication.

The leading causes of bacterial device-associated infection are Staphylococcus sp, Enterococcus faecalis, and Pseudomonas aeruginosa (Arciola et al., 2018). These pathogens vary widely in their biochemistry, antimicrobial sensitivities, and virulence mechanisms employed to cause disease. Moreover, deviceassociated infections often pose difficulties in diagnosis using classic culture techniques (Fernandez-Sampedro et al., 2017), however, new molecular methodologies have shown promise in permitting accurate pathogen identification (Costerton et al., 2011). While staphylococci are the leading cause of prosthetic joint infections (Arciola et al., 2018), the causative species of other implant-associated infections largely depend on the implant site and type, patient co-morbidities, geographical location, and time since surgery (Aggarwal et al., 2014).

Medical implants are rapidly coated with host extracellular matrix molecules that help modulate a foreign body reaction, but also represent a surface target for bacterial attachment. As opposed to the planktonic lifestyle, biofilms are adherent communities of bacteria, often encased within a self-produced matrix of DNA, proteins, and/or polysaccharides. The biofilm mode of growth is regulated by a complex network of genetic factors responding to various environmental cues, including available metabolites, host molecules, and quorum sensing (Stewart and Franklin, 2008; Arciola et al., 2012). Moreover, biofilms are notoriously recalcitrant to antibiotic therapy due to metabolic heterogeneity and are well-described for their ability to resist innate immune defenses, including leukocyte invasion and phagocytosis (Thurlow et al., 2011; Mulcahy et al., 2014; Gries and Kielian, 2017). Three-dimensional biofilm structure not only poses a physical barrier to host immune cell infiltration and phagocytosis (Gries et al., 2020), but biofilm products can actively skew immune responses to enable infection persistence (Benoit et al., 2008; Scherr et al., 2014; Gries et al., 2016).

Many in vitro and in vivo models to have been developed to study biofilm development and host immune responses (Lebeaux et al., 2013). In vitro methods often comprise of static or sheer flow models mimicking infections associated with relatively stationary (e.g., internal fixation, peripheral catheters, etc.) or dynamic (e.g., joint arthroplasty, cardiac valve, vascular catheter, etc.) sites, respectively. These models can also include the addition of host factors or immune cells to assess anti-biofilm activity and/or bacterial responses. In vivo methods examining biofilm-mediated infections often involve rodent or rabbit models of device-associated infection. These models are largely limited to ex vivo analyses; requiring sacrifice of the animal for end-point quantification, including bacteria burdens, tissue histology, flow cytometry, and/or quantifying cytokine/chemokine production. More recently, continuous monitoring of biofilm infection has been demonstrated with bioluminescent bacterial strains and fluorescent reporter animals using whole-animal in vivo imaging systems (Thurlow et al., 2011; Wang et al., 2017; Gutierrez Jauregui et al., 2019). These models are advantageous as they do not require sacrifice of the animal to glean useful data, however they are limited by camera sensitivity and associating bioluminescent image data with established bacterial burden standard curves. In addition, these small-animal imaging systems do not permit cellular-level resolution and therefore rely on large number of congregating bioluminescent or fluorescent cells to emit a detectable signal.

To assess the cellular activities and interactions occurring during biofilm infection, several studies have employed confocal or epifluorescent microscopy (Forestier et al., 2017; Abdul Hamid et al., 2020). Unfortunately, these experiments are restricted to a limited depth penetration and single shortwavelength excitatory light that rapidly damages animal tissues. Unlike confocal and epifluorescent microscopy, multiphoton microscopy (MPM) utilizes simultaneous absorption of two or more long-wavelength photons to produce a single, shortwavelength excitatory stimulus. Longer wavelengths of light enable greater tissue penetration without the damaging effects of confocal/epifluorescent light sources (Denk et al., 1990), thereby permitting time-lapse imaging within living tissues. Furthermore, multiple detectors and spectral imaging confer the ability for spatiotemporal multiplexing and second-harmonic generation. MPM technology has rapidly evolved to include high-speed laser scanning and optical sectioning with up to $1 \mathrm{~mm}$ depth penetration, allowing $3-\mathrm{D}$ reconstruction of tissue.

While MPM has proven useful in examining bacterial infection in living animals (Hickman et al., 2009; Abtin et al., 2014; Stolp and Melican, 2016), to-date MPM has not been employed to examine bacterial biofilm-mediated infections. Here we report on the use of MPM to simultaneously assess Staphylococcus aureus biofilm development and innate immune cell activity. At the time of preparation, this is the first report to examine $S$. aureus biofilm and associated innate immune response using intravital MPM. Furthermore, previous studies have shown a paucity of neutrophil influx at the site of infection (Thurlow et al., 2011; Hanke and Kielian, 2012), despite their enhanced ability to invade $S$. aureus biofilm in vitro compared to macrophages (Gunther et al., 2009; Scherr et al., 2013). We hypothesize that $S$. aureus biofilms modulate neutrophil behavior to promote infection persistence. To test this, we utilized cell tracking during time-lapse MPM to monitor neutrophil migration behavior and determined that neutrophils associated with $S$. aureus biofilm infection migrate randomly, indicating that they are redirected to avoid interaction, permitting biofilm persistence.

\section{METHODS}

\section{Bacterial Strains and Culture Conditions}

The wild-type $S$. aureus strain used in this study was LAC-13C, a USA300 MRSA skin and soft tissue infection isolate cured of plasmid p03 (Fey et al., 2013), widely used in biofilm infection studies with comparable findings to other strains (Vidlak and Kielian, 2016). The plasmid pCM29 was used as a constitutive source of superfolder-GFP (sGFP) expression (Pang et al., 2010). For infection studies, bacteria were prepared by inoculating 
freshy isolated colonies into $25 \mathrm{~mL}$ Brain-Heart Infusion broth (BHI; Oxoid, UK) containing $10 \mu \mathrm{g} \mathrm{ml}^{-21}$ chloramphenicol and cultured for $16 \mathrm{~h}$ at $37^{\circ} \mathrm{C}, 250 \mathrm{RPM}$. Cells were then washed twice with $\mathrm{PBS}$ and diluted to $5 \times 10^{5} \mathrm{CFU} \mathrm{mL}^{-1}$ prior to infection.

\section{Animals}

The wild-type C57Bl/6 and CX3CR1-EGFP knock-in mice were obtained from The Jackson Laboratory (Bar Harbor, ME, USA). The PGRP-S-DsRed mice, where DsRed expression is driven by the PGRP-S promoter (Wang et al., 2011), were bred onto the $\mathrm{C} 57 \mathrm{Bl} / 6$ background. All mice were bred in the University of California, Riverside vivarium under specific pathogen-free conditions and were handled in accordance with Institutional Animal Care and Use Committee and National Institutes of Health guidelines.

\section{Implant Fabrication}

The implant used in this study was shaped from a highconsistency medical-grade translucent silicone elastomer (MED4780; NuSil, Carpinteria, CA, USA), approved for human implantation for a period of greater than 29 days. A $2 \mathrm{~mm}$ thick section of MED-4780 was embedded in optimal cutting temperature compound (OCT; Thermo Fischer Scientific, Waltham, MA, USA) and cut at $250 \mu \mathrm{m}$ using a cryostat microtome, resulting in a $2 \times 0.25 \mathrm{~mm}$ slice. The pieces were further hand-cut to have final dimensions of $3 \times 1.0 \times 0.25 \mathrm{~mm}$ and sterilized by autoclaving.

\section{Ear Pinna Implant Infection Model}

Mice were anesthetized using ketamine-xylazine $(100 \mathrm{mg} / \mathrm{kg}-5$ $\mathrm{mg} / \mathrm{kg}$, IP; MilliporeSigma, St. Louis, MO, USA) and placed on a $36^{\circ} \mathrm{C}$ heat mat for the duration of the surgery. The left ear was affixed to a petri dish using double-sided adhesive tape and the dorsal side hair was removed using hair removal lotion. The ear was then sanitized with povidone-iodide or $70 \%$ ethanol and a small incision was made through the dorsal cutaneous layer with a scalpel. A pocket was then formed by gently separating the dermal layers of the ear pinna using a non-serrated specimen forceps. Next, $10^{3}$ CFUs of $S$. aureus in $2 \mu \mathrm{L}$ PBS was inoculated directly into the ear pocket. In some cases, the surgery and implant were kept sterile to monitor aseptic conditions. After insertion of the implant, the incision was closed using VetBond tissue adhesive (3M, Saint Paul, MN, USA).

\section{Multiphoton Microscopy (MPM)}

To assess both biofilm formation and innate immune cell activity, at least 3 sites directly on and adjacent to the biofilm/implant were imaged in each mouse. Time-lapse Z-stack images were acquired every 1-2 min over a 20-40 min span with $1 \mu \mathrm{M}$ slices (times varied due to section thickness) using a $40 \times$ water immersion lens. MPM was carried out at the UC Riverside Center for Intravital Imaging, equipped with a Nikon A1R Multiphoton Plus (MP+; Nikon, Tokyo, Japan) microscope, including an auto-aligned tunable $(700-1,080 \mathrm{~nm})$ infrared laser (Coherent, Santa Clara, CA, USA), resonant and galvano scanners, 3 Gallium-arsenide-phosphide (GaAsP) non-descanned detectors
(NDD), and 1 high sensitivity NDD for IR detection. The Nikon $\mathrm{A} 1 \mathrm{R} \mathrm{MP}+$ is housed in a procedure room within the specific pathogen-free rodent vivarium and was fitted for BSL-2 usage. Still images and movies were collected, analyzed, and prepared for publication using Nikon NIS-Elements software.

\section{Cell Tracking and Quantification}

Neutrophil migration patterns were quantified using Volocity software (Quorum Technologies, Guelph, Ontario, Canada). Automated tracking algorithms to follow cells was confirmed using manual tracking by marking each individual object at each timepoint. The measurements of each neutrophil at each timepoint was taken relative to the centroid of the region of interest and were collectively gathered to form one measurement. Several parameters to define cell behavior were assessed, including velocity, displacement, and meandering index. Velocity represents the average speed of the neutrophil over the track. Displacement represents the average straight line distance from the first timepoint centroid to the last. Meandering index measures deviation from a straight line, with values from 0 to 1 .

\section{Post-infection Analyses}

After 9 days, animals were sacrificed, and the infected ear was collected for bacterial burden enumeration associated with the implant and surrounding soft tissue. Briefly, the ear was removed, rinsed with $70 \%$ ethanol, and the excised implant placed in 100 $\mu \mathrm{L}$ PBS for sonication to dissociate bacteria from the implant surface. The ear was then weighed, cut into smaller pieces, then dissociated in $500 \mu \mathrm{L}$ of PBS using the blunt end of a $3 \mathrm{~mL}$ syringe plunger. Tissue and implant bacterial titers were quantified on TSA and expressed as CFU per $\mathrm{mL}$ for implants and CFU per gram for tissue.

\section{Statistical Analyses}

Significant differences between experimental groups were determined as described in the respective figure legends. GraphPad Prism 8 (GraphPad, San Diego, CA, USA) was used for all statistical analysis calculations, and a $P<0.05$ was considered statistically significant. Biofilm images are representative from 8 independent experiments. Neutrophil tracking data are from two independent experiments with statistical analyses performed using an unpaired, two-tailed $t$-test.

\section{RESULTS}

The mouse ear pinna is a proven site for assessing immune responses and infection progression (Li et al., 2012; Abtin et al., 2014; Forestier et al., 2017). Thus, we sought to establish an ear pinna interdermal implant model of $S$. aureus biofilm infection. Briefly, for the implant surgery and infection, the left ear of an anesthetized mouse was affixed to a petri dish and the dorsal side hair removed (Figures 1A,B). The ear was then sanitized, a small incision made through the outer cutaneous layer, and a small pocket formed by gently separating the dermal layers of the ear pinna (Figures 1C,D). Next, $10^{3}$ CFU of $S$. aureus LAC13C harboring pCM29 was pipetted directly into the interdermal 

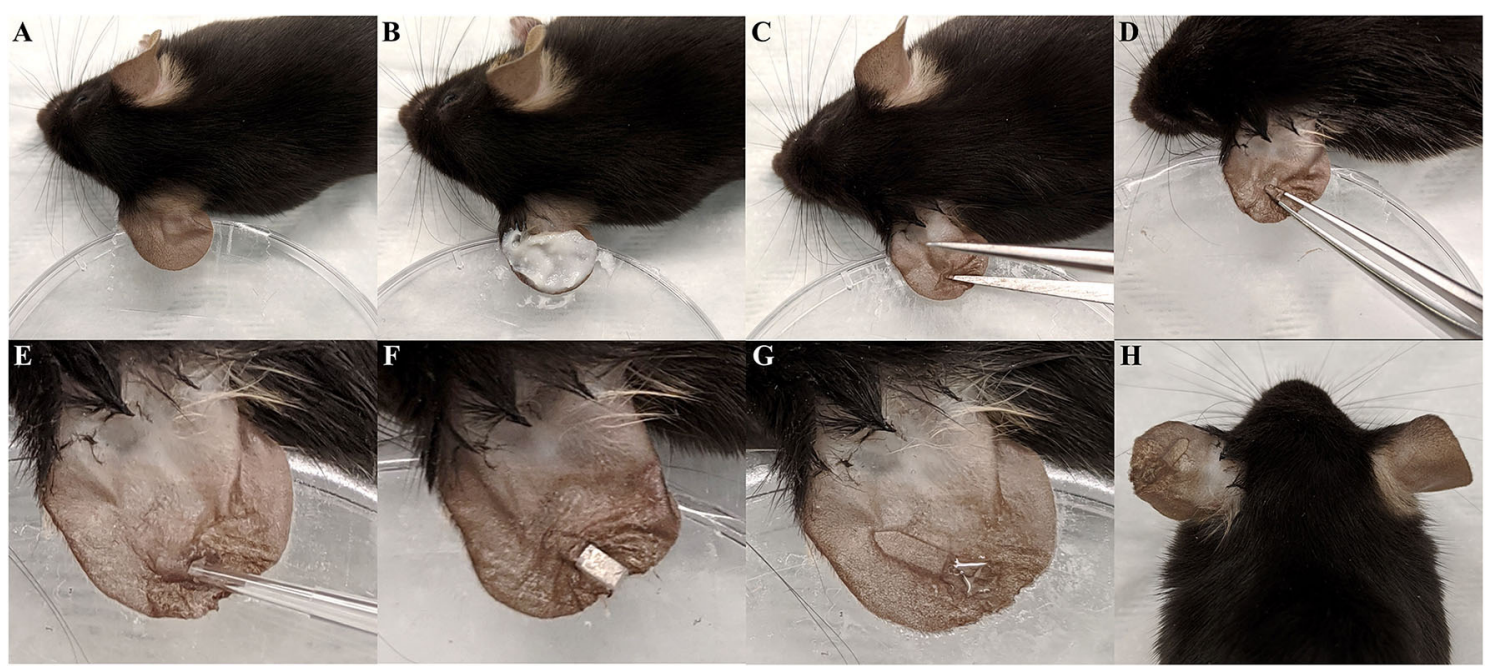

FIGURE 1 | Interdermal implant -associated biofilm infection model surgery. (A) The left ear pinna of an anesthetized mouse was affixed to a petri dished using double-sided tape. (B) Dorsal side hair was removed from the ear using hair removal cream. (C,D) A small incision was made in the dorsal side dermis and widened into a pocket using forceps. (E) $10^{3} \mathrm{CFU}$ of $S$. aureus in $2 \mu \mathrm{L}$ PBS was then inoculated into the pinna pocket. (F) The $0.25 \mathrm{~mm}$ thin slice of silicone elastomer was slid into the pocket. (G) The incision was closed with tissue adhesive. (H) Final appearance of the ear following surgery and infection.
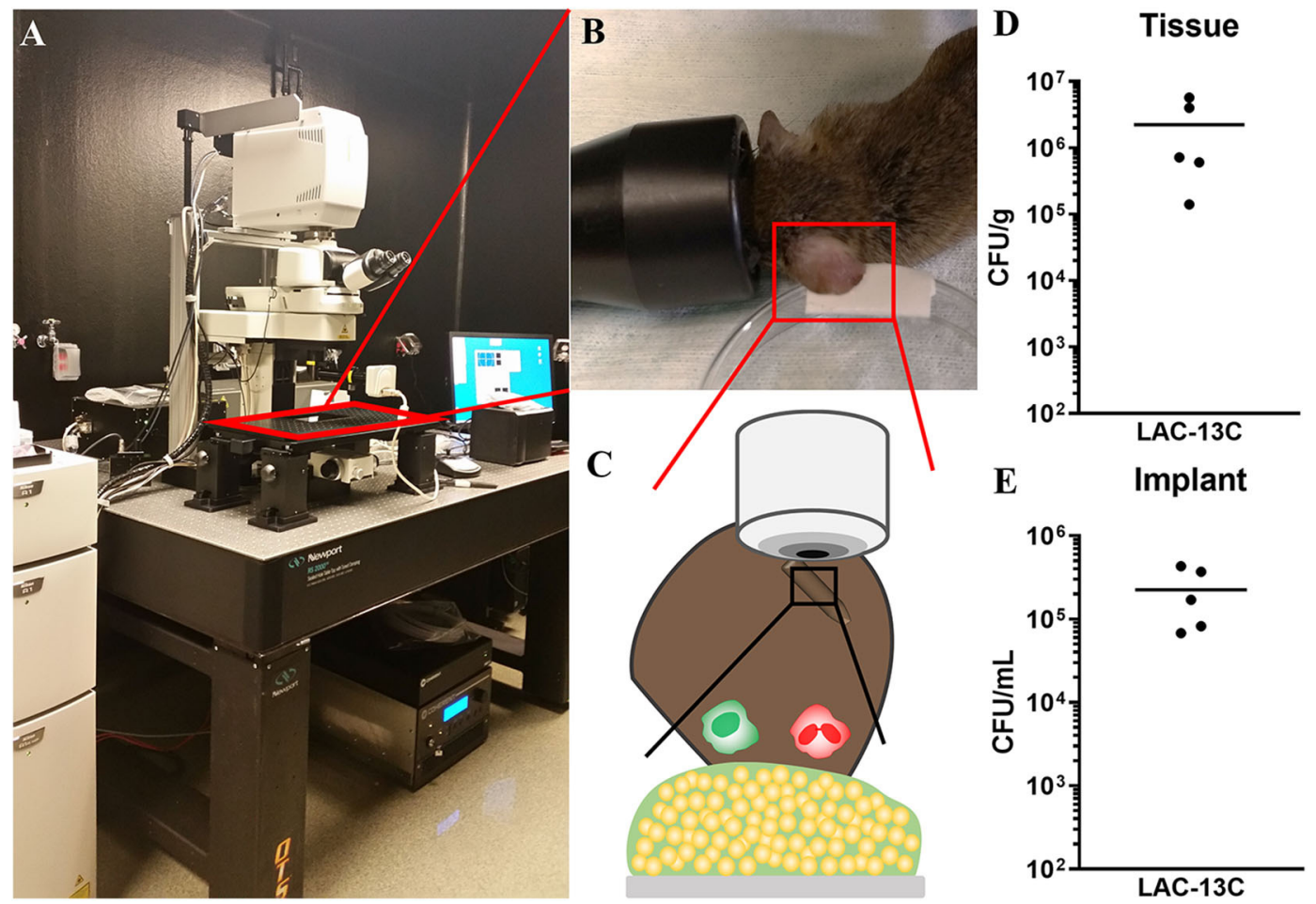

FIGURE 2 | Multiphoton microscopy illustration and post-infection analysis of implant-associated S. aureus biofilm infection. (A-C) MPM arrangement with a cartoon illustration of biofilm infection visualization. (D,E) Day 9 post-infection analysis of bacterial burdens associated with surrounding ear tissue (D) and the silicone implant (E). Data are from two independent experiments with the horizontal line representing the mean.

pocket (Figure 1E). Finally, a section of translucent medical grade silicone was then inserted into the ear pinna pocket and the incision closed using tissue adhesive (Figures 1F-H).
Murine device-associated S. aureus biofilm infections typically reach maximum bacterial burdens 3 days post-infection, with bona fide biofilms formed by day 7 (Thurlow et al., 2011; Heim 

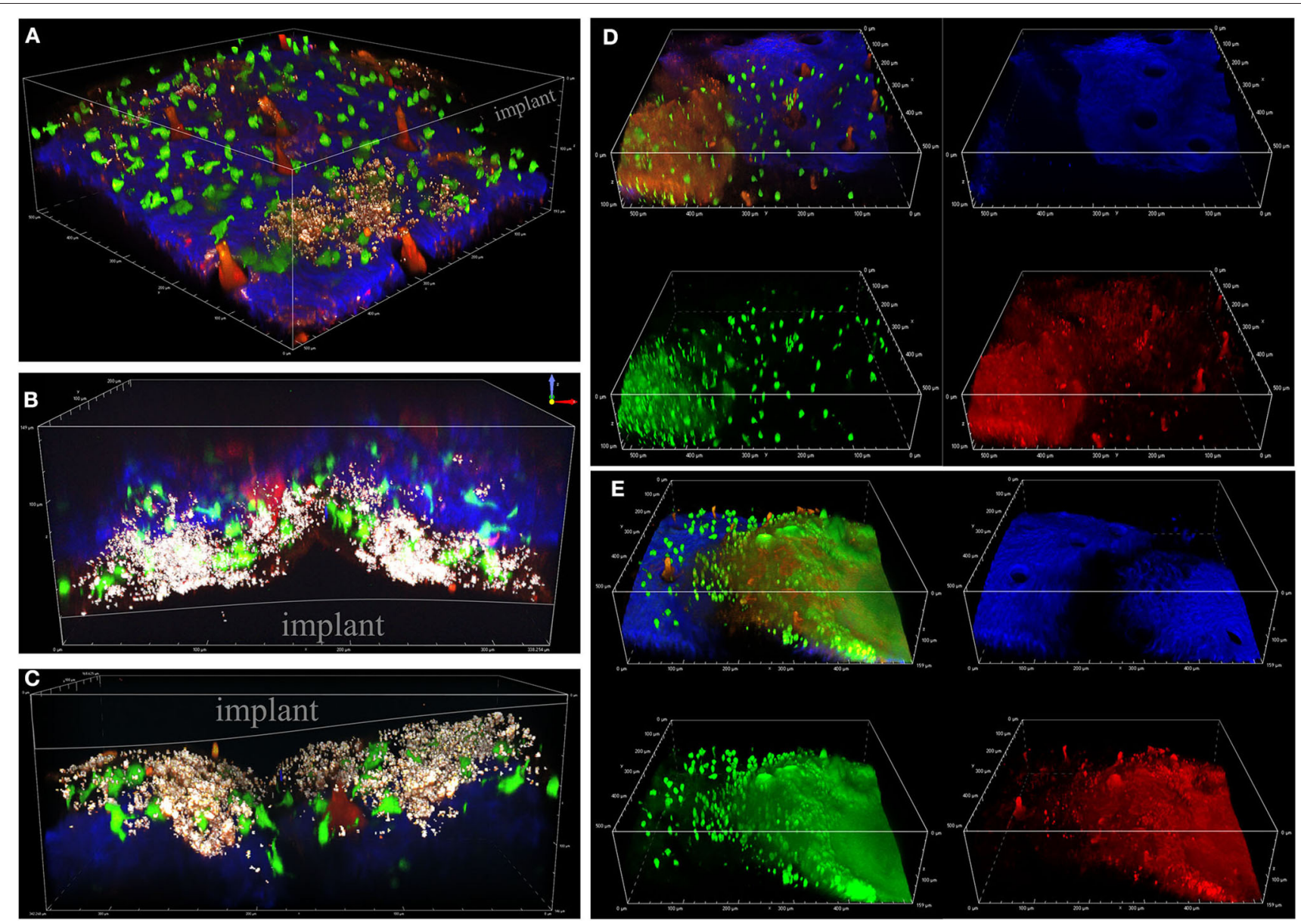

FIGURE 3 | Visualizing S. aureus biofilm infection and immune cell interactions. Intravital MPM of a CX3CR1-EGFP reporter mouse infected with S. aureus, shown in bright white. EGFP ${ }^{+}$macrophages/dendritic cells are shown in green, and second-harmonic generated connective tissue (blue) and vasculature (red). Bacterial cells are depicted in red-white as superfolder-GFP expression is detectable in all channels. Representative images depict biofilms at 3 (A), 5 (B,C), 7 (D), and 9 (E) days post-infection, taken from 8 independent experiments.

et al., 2015; Yamada et al., 2018). Thus, intravital MPM imaging took place daily starting 2 days and ending 9 days post-infection (Figures 2A-C). Importantly, this model and time frame permits the establishment of a robust infection associated with both the implant and surrounding tissue. As shown in Figures 2D,E, tissue and implant-associated bacterial burdens after 9 days were comparable to those observed in other $S$. aureus biofilm infection models (Yamada et al., 2018; Gries et al., 2020).

To visualize $S$. aureus biofilm development and associated innate immune activity in vivo, we utilized a CX3CR1-EGFP transgenic mouse where EGFP is expressed in monocytes and dendritic cells (Jung et al., 2000). Representative images of $S$. aureus biofilm development over 9 days post-infection are shown in Figure 3. S. aureus cells are shown in red-white as the bright superfolder GFP emitted fluorescence in both the FITC and TRITC channels. The early stages of infection (days 3 and 5) depict a loose accumulation of individually visible $S$. aureus cells associated with the implant (Figures 3A-C). Later stages of infection (days 7 and 9) revealed a larger mass of cells encased in a hazy matrix that largely prevented the visualization of individual bacterial cells (Figures 3D,E). While the composition of this extracellular matrix remains to be determined, it is possible that this is the result of mature biofilm matrix development.

To measure neutrophil migration patterns in response to sterile vs. biofilm infected implants, we utilized a PGRPS-DsRed transgenic reporter mouse. The tracking paths of neutrophils associated with sterile and infected tissues on day 3 post-surgery are show in Figures 4A,B. Importantly, neutrophils near but not in direct contact with individual bacterial cells or the biofilm were assessed. Compared to neutrophils associated with a sterile implant, those near the infected implant showed significantly increased cell migration velocity and displacement (Figures 4C,D). Interestingly, these patterns were also associated with a significantly increased meandering index, or what appeared to be rapid but aimless neutrophil migration (Figure 4E). Together, these data shed 


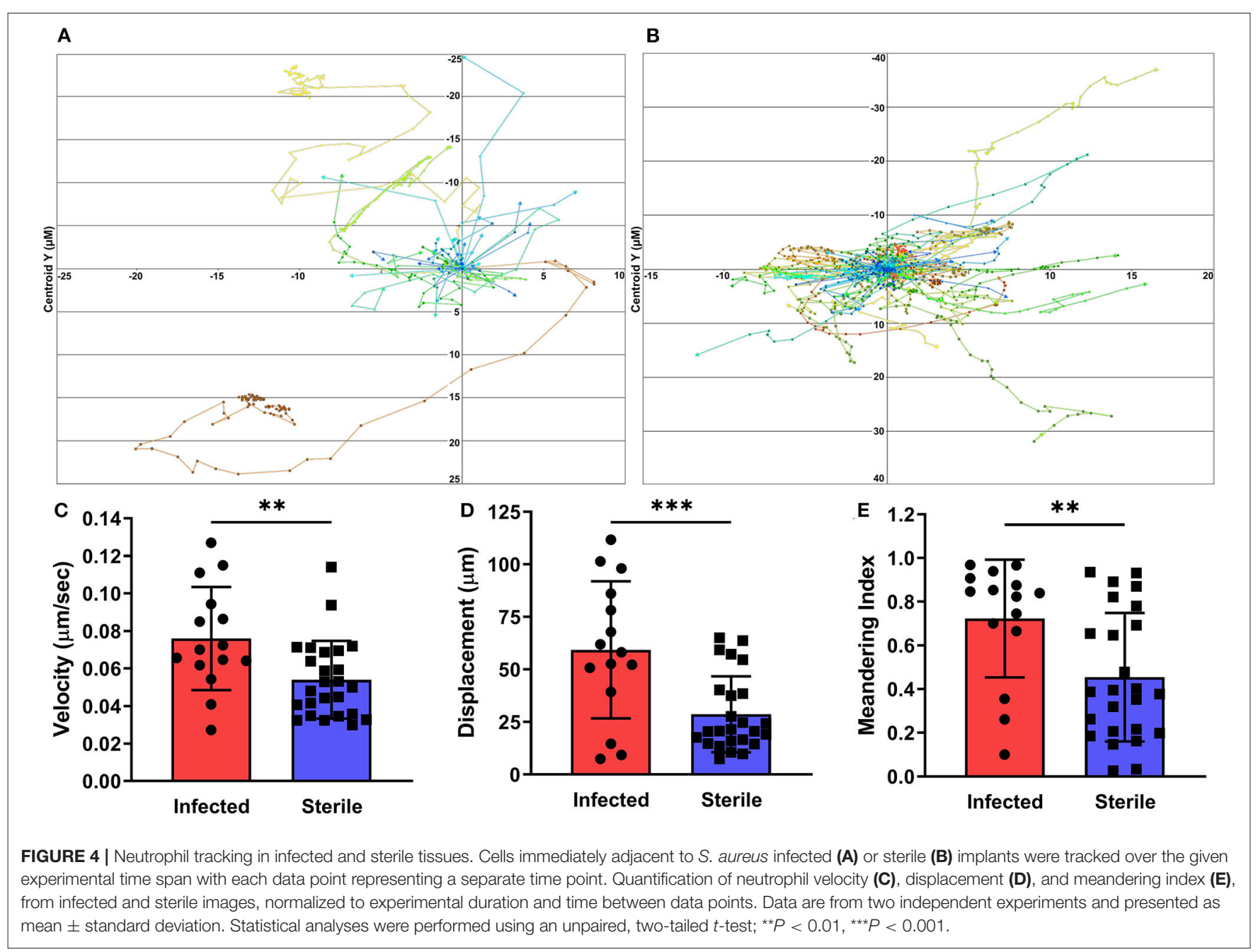

light on the development of S. aureus biofilm during deviceassociated infection and the effects of the biofilm infection on neutrophil activity.

\section{DISCUSSION}

Staphylococcus sp. biofilms are the most common cause of medical device-associated infections (Arciola et al., 2018). These infections can arise in otherwise healthy individuals and often lead to chronic, reoccurring infections. Productive innate immune responses are inhibited by $S$. aureus biofilm products, including polarizing infiltrating macrophages and recruitment of myeloid-derived suppressor cells (Scherr et al., 2015; Gries and Kielian, 2017). Moreover, the three-dimensional biofilm architecture itself presents a barrier to immune cell infiltration and phagocytosis in vitro (Thurlow et al., 2011; Gries et al., 2020). However, relatively little is known of the hostpathogen interface during $S$. aureus biofilm-mediated infection. In this work we demonstrate the capability of multiphoton microscopy (MPM) to assess bacterial biofilm development and measure associated neutrophil migratory activity. To date, this is the first report using MPM to assess device-associated bacterial biofilm-mediated infection and concomitant innate immune responses.

The mouse ear pinna presents an ideal tissue to examine biofilm development and is a proven site to analyze innate immune activity using MPM (Li et al., 2012). Relative to the mouse body, the ear is sparse in auto-fluorescent hair coverage which can be easily removed prior to surgery or imaging. Furthermore, the implant/infection surgery causes minimal tissue damage and requires few specialized tools. Finally, MPM of the ear pinna is non-invasive and permits extended timelapse imaging over multiple days using the same animal. In our model, the CX3CR1-EGFP and PGRP-S-DsRed transgenic animals expedite the orientation and focus within the pinna tissue. The $S$. aureus superfolder GFP used in these studies is highly fluorescent and easily detectable in both the FITC and TRITC channels. While this may present some future challenges, it allows easy detection of bacterial cells in the context of second harmonics and mouse fluorescent reporter expression. Notably, 
we did not measure any significant loss of the GFP plasmid over the 9-day period (data not shown).

Using MPM, we sought to examine the developmental stages of $S$. aureus biofilm formation in vivo and simultaneously examine innate immune cell responses. This is a necessary step as multiple in vitro models of biofilm/immune cell co-culture have been employed, but their accuracy to biofilm infection has not been well-established. While many device-associated infections are multispecies, we limited this study to assess $S$. aureus biofilms and their impact on innate immune cell function. We showed that $S$. aureus device-associated infections follow a developmental progression and time frame analogous to that observed in vitro using minimal media such as RPMI (Thurlow et al., 2011; Gries et al., 2016). Initially, single S. aureus cells are easily observable and closely associated with the implant. By day 5, the number of visible bacterial cells was markedly increased. It remains unclear why maximum bacterial burdens are typically seen by day 3 post-infection, yet a considerable difference exists in the number of bacteria observed between days 3 and 5. A possible explanation is that bacterial viability was substantially decreased between these time points, however further examination of viability would be required to delineate this observation.

Mature S. aureus biofilms are characterized as having an extensive extracellular matrix composed of various proteins, sugars, and extracellular DNA (Foulston et al., 2014; Lister and Horswill, 2014). It was therefore not surprising to find that after 7 days, an extensive, hazy covering of the bacterial cells appeared. The matrix would also likely consist of a significant portion of GFP molecules, potentially providing its robust fluorescent appearance. An interesting observation throughout the time course is the overall lack of phagocytosis by monocytes, dendritic cells, and neutrophils. While this is in agreement with in vitro findings (Thurlow et al., 2011; Gries et al., 2020), a paucity of phagocytosis was observed beginning on day 3 postinfection, before the robust biofilm structure was formed. Further investigation will be required to assess the role of $S$. aureus biofilm molecules known to affect macrophage and neutrophil phagocytic function (Scherr et al., 2015; Gries et al., 2016; Bhattacharya et al., 2018).

Neutrophils and macrophages are essential cellular components of the innate immune response to bacterial biofilm infection. Their presence also markedly alters $S$. aureus biofilm gene expression in vitro (Scherr et al., 2013). Neutrophils are the first line of cellular immune defense against

\section{REFERENCES}

Abdul Hamid, A. I., Nakusi, L., Givskov, M., Chang, Y. T., Marques, C., and Gueirard, P. (2020). A mouse ear skin model to study the dynamics of innate immune responses against Staphylococcus aureus biofilms. BMC Microbiol. 20:22. doi: 10.1186/s12866-019-1635-Z

Abtin, A., Jain, R., Mitchell, A. J., Roediger, B., Brzoska, A. J., Tikoo, S., et al. (2014). Perivascular macrophages mediate neutrophil recruitment during bacterial skin infection. Nat. Immunol. 15, 45-53. doi: 10.1038/ ni. 2769 invading bacterial pathogens; their coordinated recruitment and activity are essential to preventing and eliminating infection. Compared to macrophages, neutrophils have shown a much higher propensity for biofilm invasion and phagocytosis in vitro (Gunther et al., 2009; Thurlow et al., 2011; Ghimire et al., 2019). We observed significant differences in the cell migration behaviors of neutrophils in response to infected versus sterile implants. Despite neutrophils appearing to respond to biofilm infection by increasing migration velocity and displacement, their movements were less directed toward the implant/infection than neutrophils in sterile tissues. These observation are consistent with a recent report that a delay in neutrophil recruitment to the implant surface may permit $S$. aureus time to grow and form biofilm (Ghimire et al., 2019). We anticipate that neutrophil deviation is a result of $S$. aureus biofilm products interfering with neutrophil cytokine/chemokine signaling and the production of multiple leukocyte inhibitors and toxins (Rooijakkers et al., 2006). The mechanism(s) responsible for neutrophil redirection away from $S$. aureus biofilm are subject of future investigations. Together, these observations further establish the ineffective nature of neutrophil responses to $S$. aureus biofilm-mediated infection.

\section{DATA AVAILABILITY STATEMENT}

The raw data supporting the conclusions of this article will be made available by the authors, without undue reservation.

\section{ETHICS STATEMENT}

The animal study was reviewed and approved by Institutional Animal Care and Use Committee.

\section{AUTHOR CONTRIBUTIONS}

CG and DL conceived and designed the experiments. CG, ZR, and JC performed the experiments. CG, ZR, JC, and DL analyzed data. CG wrote the paper. All authors contributed to the article and approved the submitted version.

\section{FUNDING}

Research reported in this publication was supported by the Research Infrastructure Programs of the National Institutes of Health under award number 1S10OD020042-01. 
A review of molecular mechanisms and implications for biofilm-resistant materials. Biomaterials 33, 5967-5982. doi: 10.1016/j.biomaterials.2012. 05.031

Benoit, M., Desnues, B., and Mege, J.L. (2008). Macrophage polarization in bacterial infections. J. Immunol. 181, 3733-3739. doi: 10.4049/jimmunol.181.6.3733

Bhattacharya, M., Berends, E. T. M., Chan, R., Schwab, E., Roy, S., Sen, C. K., et al. (2018). Staphylococcus aureus biofilms release leukocidins to elicit extracellular trap formation and evade neutrophil-mediated killing. Proc. Natl. Acad. Sci. U. S. A. 115, 7416-7421. doi: 10.1073/pnas. 1721949115

Costerton, J. W., Montanaro, L., and Arciola, C. R. (2005). Biofilm in implant infections: its production and regulation. Int. J. Artif. Organs. 28, 1062-1068. doi: $10.1177 / 039139880502801103$

Costerton, J. W., Post, J. C., Ehrlich, G. D., Hu, F. Z., Kreft, R., Nistico, L., et al. (2011). New methods for the detection of orthopedic and other biofilm infections. FEMS Immunol. Med. Microbiol. 61, 133-140. doi: 10.1111/j.1574-695X.2010.00766.x

Denk, W., Strickler, J. H., and Webb, W. W. (1990). Two-photon laser scanning fluorescence microscopy. Science 248, 73-76. doi: 10.1126/science. 2321027

Fernandez-Sampedro, M., Farinas-Alvarez, C., Garces-Zarzalejo, C., Alonso-Aguirre, M. A., Salas-Venero, C., Martinez-Martinez, L., et al. (2017). Accuracy of different diagnostic tests for early, delayed and late prosthetic joint infection. BMC Infect. Dis. 17:592. doi: 10.1186/ s12879-017-2693-1

Fey, P. D., Endres, J. L., Yajjala, V. K., Widhelm, T. J., Boissy, R. J., Bose, J. L., et al. (2013). A genetic resource for rapid and comprehensive phenotype screening of nonessential Staphylococcus aureus genes. Mbio 4, e00537-e00512. doi: 10.1128/mBio.00537-12

Forestier, C., Billard, E., Milon, G., and Gueirard, P. (2017). Unveiling and characterizing early bilateral interactions between biofilm and the mouse innate immune system. Front. Microbiol. 8:2309. doi: 10.3389/fmicb.2017. 02309

Foulston, L., Elsholz, A. K., Defrancesco, A. S., and Losick, R. (2014). The extracellular matrix of Staphylococcus aureus biofilms comprises cytoplasmic proteins that associate with the cell surface in response to decreasing $\mathrm{pH}$. Mbio 5, e01667-e01614. doi: 10.1128/mBio.01667-14

Ghimire, N., Pettygrove, B. A., Pallister, K. B., Stangeland, J., Stanhope, S., Klapper, I., et al. (2019). Direct microscopic observation of human neutrophilStaphylococcus aureus interaction in vitro suggests a potential mechanism for initiation of biofilm infection on an implanted medical device. Infect. Immun. 87, e00745-19. doi: 10.1128/IAI.00745-19

Gries, C. M., Biddle, T., Bose, J. L., Kielian, T., and Lo, D. D. (2020). Staphylococcus aureus fibronectin binding protein A mediates biofilm development and infection. Infect. Immun. 6, e00413-15. doi: 10.1128/IAI. 00859-19

Gries, C. M., Bruger, E. L., Moormeier, D. E., Scherr, T. D., Waters, C. M., and Kielian, T. (2016). Cyclic di-AMP released from Staphylococcus aureus biofilm induces a macrophage type I interferon response. Infect. Immun. 84, 3564-3574. doi: 10.1128/IAI.00447-16

Gries, C. M., and Kielian, T. (2017). Staphylococcal biofilms and immune polarization during prosthetic joint infection. J. Am. Acad. Orthop. Surg. 25 (Suppl. 1), S20-S24. doi: 10.5435/JAAOS-D-16-00636

Gunther, F., Wabnitz, G. H., Stroh, P., Prior, B., Obst, U., Samstag, Y., et al. (2009). Host defence against Staphylococcus aureus biofilms infection: phagocytosis of biofilms by polymorphonuclear neutrophils (PMN). Mol. Immunol. 46, 1805-1813. doi: 10.1016/j.molimm.2009. 01.020

Gutierrez Jauregui, R., Fleige, H., Bubke, A., Rohde, M., Weiss, S., and Forster, R. (2019). IL-1 $\beta$ promotes Staphylococcus aureus biofilms on implants in vivo. Front. Immunol. 10:1082. doi: 10.3389/fimmu.2019. 01082

Hanke, M. L., and Kielian, T. (2012). Deciphering mechanisms of staphylococcal biofilm evasion of host immunity. Front. Cell. Infect. Microbiol. 2:62. doi: $10.3389 /$ fcimb.2012.00062

Heim, C. E., Vidlak, D., and Kielian, T. (2015). Interleukin-10 production by myeloid-derived suppressor cells contributes to bacterial persistence during
Staphylococcus aureus orthopedic biofilm infection. J. Leukoc. Biol. 98, 1003-1013. doi: 10.1189/jlb.4VMA0315-125RR

Hickman, H. D., Bennink, J. R., and Yewdell, J. W. (2009). Caught in the act: intravital multiphoton microscopy of host-pathogen interactions. Cell Host Microbe 5, 13-21. doi: 10.1016/j.chom.2008. 12.007

Jung, S., Aliberti, J., Graemmel, P., Sunshine, M. J., Kreutzberg, G. W., Sher, A., et al. (2000). Analysis of fractalkine receptor CX(3)CR1 function by targeted deletion and green fluorescent protein reporter gene insertion. Mol. Cell. Biol. 20, 4106-4114. doi: 10.1128/MCB.20.11.4106-4114. 2000

Kurtz, S., Ong, K., Lau, E., Mowat, F., and Halpern, M. (2007). Projections of primary and revision hip and knee arthroplasty in the United States from 2005 to 2030. J. Bone Joint Surg. Am. 89, 780-785. doi: 10.2106/00004623-20070400000012

Kurtz, S. M., Lau, E., Watson, H., Schmier, J. K., and Parvizi, J. (2012). Economic burden of periprosthetic joint infection in the United States. J. Arthroplasty. 27, 61-65.e61. doi: 10.1016/j.arth.2012.02.022

Lebeaux, D., Chauhan, A., Rendueles, O., and Beloin, C. (2013). From in vitro to in vivo models of bacterial biofilm-related infections. Pathogens 2, 288-356. doi: $10.3390 /$ pathogens 2020288

Li, J. L., Goh, C. C., Keeble, J. L., Qin, J. S., Roediger, B., Jain, R., et al. (2012). Intravital multiphoton imaging of immune responses in the mouse ear skin. Nat. Protoc. 7, 221-234. doi: 10.1038/nprot. 2011.438

Lister, J. L., and Horswill, A. R. (2014). Staphylococcus aureus biofilms: recent developments in biofilm dispersal. Front. Cell. Infect. Microbiol. 4:178. doi: $10.3389 /$ fcimb.2014.00178

Magill, S. S., Edwards, J. R., Bamberg, W., Beldavs, Z. G., Dumyati, G., Kainer, M. A., et al. (2014). Multistate point-prevalence survey of health care-associated infections. N. Engl. J. Med. 370, 1198-1208. doi: 10.1056/ NEJMoal306801

Mulcahy, L. R., Isabella, V. M., and Lewis, K. (2014). Pseudomonas aeruginosa biofilms in disease. Microb. Ecol. 68, 1-12. doi: 10.1007/ s00248-013-0297-x

Pang, Y. Y., Schwartz, J., Thoendel, M., Ackermann, L. W., Horswill, A. R., and Nauseef, W. M. (2010). agr-dependent interactions of Staphylococcus aureus USA300 with human polymorphonuclear neutrophils. J. Innate. Immun. 2, 546-559. doi: 10.1159/000319855

Rooijakkers, S. H., Ruyken, M., Van Roon, J., Van Kessel, K. P., Van Strijp, J. A., and Van Wamel, W. J. (2006). Early expression of SCIN and CHIPS drives instant immune evasion by Staphylococcus aureus. Cell. Microbiol. 8, 1282-1293. doi: 10.1111/j.1462-5822.2006. 00709.x

Scherr, T. D., Hanke, M. L., Huang, O., James, D. B., Horswill, A. R., Bayles, K. W., et al. (2015). Staphylococcus aureus biofilms induce macrophage dysfunction through leukocidin $\mathrm{AB}$ and alpha-toxin. mBio 6, e01021-15. doi: 10.1128/mBio.01021-15

Scherr, T. D., Heim, C. E., Morrison, J. M., and Kielian, T. (2014). Hiding in plain sight: interplay between staphylococcal biofilms and host immunity. Front. Immunol. 5:37. doi: 10.3389/fimmu.2014.00037

Scherr, T. D., Roux, C. M., Hanke, M. L., Angle, A., Dunman, P. M., and Kielian, T. (2013). Global transcriptome analysis of Staphylococcus aureus biofilms in response to innate immune cells. Infect. Immun. 81, 4363-4376. doi: 10.1128/IAI.00819-13

Stewart, P. S., and Franklin, M. J. (2008). Physiological heterogeneity in biofilms. Nat. Rev. Microbiol. 6, 199-210. doi: 10.1038/ nrmicro1838

Stolp, B., and Melican, K. (2016). Microbial pathogenesis revealed by intravital microscopy: pros, cons and cautions. FEBS Lett. 590, 2014-2026. doi: $10.1002 / 1873-3468.12122$

Tande, A.J., and Patel, R. (2014). Prosthetic joint infection. Clin. Microbiol. Rev. 27, 302-345. doi: 10.1128/CMR.00111-13

Thurlow, L. R., Hanke, M. L., Fritz, T., Angle, A., Aldrich, A., Williams, S. H., et al. (2011). Staphylococcus aureus biofilms prevent macrophage phagocytosis and attenuate inflammation in vivo. J. Immunol. 186, 6585-6596. doi: 10.4049/jimmunol. 1002794 
Vidlak, D., and Kielian, T. (2016). Infectious dose dictates the host response during Staphylococcus aureus orthopedic-implant biofilm infection. Infect. Immun. 84, 1957-1965. doi: 10.1128/IAI.00117-16

Wang, J., Gusti, V., Saraswati, A., and Lo, D. D. (2011). Convergent and divergent development among $\mathrm{M}$ cell lineages in mouse mucosal epithelium. J. Immunol. 187, 5277-5285. doi: 10.4049/jimmunol. 1102077

Wang, Y., Cheng, L. I., Helfer, D. R., Ashbaugh, A. G., Miller, R. J., Tzomides, A. J., et al. (2017). Mouse model of hematogenous implantrelated Staphylococcus aureus biofilm infection reveals therapeutic targets. Proc. Natl. Acad. Sci. U. S. A. 114, E5094-E5102. doi: 10.1073/pnas. 1703427114

Yamada, K. J., Heim, C. E., Aldrich, A. L., Gries, C. M., Staudacher, A. G., and Kielian, T. (2018). Arginase-1 expression in myeloid cells regulates
Staphylococcus aureus planktonic but not biofilm infection. Infect. Immun. 86, e00206-18. doi: 10.1128/IAI.00206-18

Conflict of Interest: The authors declare that the research was conducted in the absence of any commercial or financial relationships that could be construed as a potential conflict of interest.

Copyright (c) 2020 Gries, Rivas, Chen and Lo. This is an open-access article distributed under the terms of the Creative Commons Attribution License (CC BY).

The use, distribution or reproduction in other forums is permitted, provided the original author(s) and the copyright owner(s) are credited and that the original publication in this journal is cited, in accordance with accepted academic practice. No use, distribution or reproduction is permitted which does not comply with these terms. 